\title{
Complete Reversion of Familial Adenomatous Polyposis Phenotype Associated with Tacrolimus and Mycophenolate Mofetil Treatment Following Kidney Transplantation
}

\author{
VERED PINSK ${ }^{1,2,3^{*}}$, ILIA PINSK ${ }^{3,4^{*}}$, GALINA LING $^{2,3,5}$, BARUCH YERUSHALMI ${ }^{1,2,3}$, \\ LIDIA OSYNTSOV ${ }^{3,6}$ and EDUARD LING ${ }^{3,7}$ \\ ${ }^{1}$ Pediatric Ambulatory Service, ${ }^{2}$ Pediatric Gastroenterology and Nutrition Unit, \\ ${ }^{5}$ Pediatrics Department $D,{ }^{7}$ Pediatrics Department B, Saban Pediatric Medical Center for Israel, \\ Soroka University Medical Center, Beer Sheva, Israel; \\ ${ }^{3}$ Faculty of Health Sciences, Ben-Gurion University of the Negev, Beer Sheva, Israel; \\ ${ }^{4}$ Colorectal Surgery Unit, Surgery Department B and ${ }^{6}$ Institute for Pathology, \\ Soroka University Medical Center, Beer Sheva, Israel
}

\begin{abstract}
Numerous germline mutations in the adenomatous polyposis coli (APC) tumor-suppressor gene are responsible for development of multiple adenomatous colorectal polyps with their inevitable progression to cancer. Multiple attempts at dietary and pharmacological prevention of colorectal carcinoma development in patients with familial adenomatous polyposis (FAP) have provided conflicting results. Immunosuppressive treatment with tacrolimus is known to be associated with an increased risk of malignancy and should be avoided in patients with high propensity for development of neoplasia. We observed a complete reversion of FAP phenotype in a male teenager carrying a germline mutation in APC gene who underwent a kidney transplant due to end-stage kidney disease secondary to congenital dysplastic kidneys. The patient was treated with tacrolimus and mycophenolate mofetil after transplantation. The possible chemopreventative role of these agents should be evaluated and confirmed in a larger cohort. The elucidation of molecular mechanisms underpinning the observed chemopreventative effect of tacrolimus and mycophenolate mofetil might lead to the development of a novel colorectal cancer therapy.
\end{abstract}

*These Authors contributed equally to this study.

Correspondence to: Dr. Eduard Ling, MD, Ph.D., Pediatrics Department B, Saban Pediatric Medical Center for Israel, Soroka University Medical Center, Rager Avenue, Beer Sheva, Israel. Tel: +972 86400755, Fax: +972 86287163, e-mail: ling@bgu.ac.il

Key Words: Polyp, regression, Wnt, apoptosis, case report.
Familial adenomatous polyposis (FAP) is a rare autosomaldominant disorder that stems from loss-of-function mutation in the adenomatous polyposis coli $(A P C)$ gene. The wild-type tumor-suppressor $A P C$ gene encodes a large polypeptide that is a part of the APC-axin-glycogen synthetase kinase $3 \beta$ complex in the cell cytoplasm, which functions to phosphorylate $\beta$-catenin, targeting the latter for degradation in the proteasome. In the absence of functional APC protein, unphosphorylated $\beta$-catenin accumulates in the cytoplasm, and is translocated to the nucleus, where it couples with members of TCF/LEF transcription factors in order to activate transcription of WNT pathway target genes, namely matrilysin, cyclin D1, and T-lymphoma invasion and metastasis-inducing protein TIAM1. This can result in development of multiple polyps in the colon of patients, sometimes with virtual replacement of colonic mucosa with hundreds and thousands of polyps (1-3). It should be noted that an alternative, $\beta$-catenin-independent WNT signaling pathway, termed non-canonical WNT, exists (3). Polyps in patients with FAP inevitably progress to adenocarcinomas. Management of FAP includes annual colonoscopy, starting at puberty, with removal of polyps, and can require prophylactic colectomy (2).

Although a plethora of drugs and dietary supplements has been implicated in chemoprevention of colon cancer, their use is not endorsed by existing guidelines (4-6).

We herein describe a case of regression of colonic polyps in a teenager with FAP who was treated with tacrolimus and mycophenolate mofetil following kidney transplantation and discuss the possible mode of chemopreventative action of these medications in colorectal cancer. 


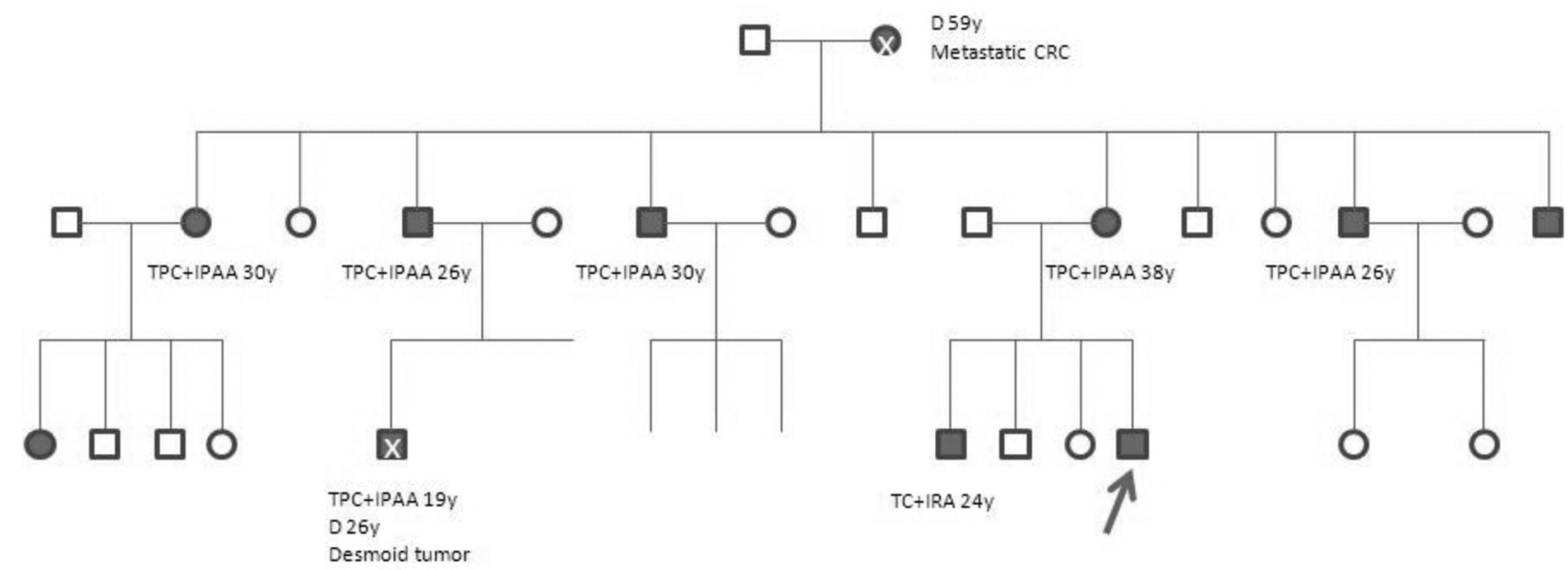

Figure 1. Pedigree of patient's family. Closed symbols, FAP. Arrow indicates the index case. CRC: Colorectal cancer, TPC: total proctocolecectomy, IPAA: ileal pouch anastomosis, IRA: ileorectal anastomosis, D: death, y: years of age.

\section{Case Report}

A 19-year-old patient of Sephardic Jewish origin was diagnosed with attenuated FAP at the age of 13 years. The genetic test, which revealed c.2520-2521delCT(het) STOP deletion mutation in codon 15 of the $A P C$ gene, was performed due to the family history of FAP. The patient's grandmother died from metastatic adenocarcinoma of the colon at the age of 59 years. Ten family members had undergone colectomy due to multiple polyps at various ages (range $=17-38$ years) and one of them had died from desmoid tumor at the age of 26 years, 6 years after colectomy (Figure 1).

The first screening colonoscopy of the patient under observation was performed at 13 years of age and was negative for colonic polyps. However, the next colonoscopy two years later, revealed 10 adenomatous polyps that were removed during colonoscopy. Histology of the resected polyp showed fragments of colonic mucosa with adenomatous changes and low-grade dysplasia (Figure 2A). The next colonoscopy, performed a year later, at the age of 16 years, revealed five additional tubular adenomas and low-grade dysplasia of mucosa. It should be noted that in addition to FAP, our patient suffered from end-stage renal disease due to congenital dysplastic kidneys and at the age of 16 years underwent kidney transplantation. Following transplantation, the patient was treated daily with $2 \mathrm{mg}$ tacrolimus, $750 \mathrm{mg}$ mycophenolate mofetil (MMF) and $5 \mathrm{mg}$ prednisone. Subsequent colonoscopies performed 6 and 14 months after kidney transplantation revealed 10 and three small polyps, respectively, which were removed. Six months later, during the next colonoscopy, there were no macroscopically detectable polyps, but histological analysis of multiple randomized biopsies of colonic mucosa showed adenomatous changes in 5 of 20 biopsies. Eight months later, adenomatous changes were found only in one crypt from 20 randomized biopsies of normal-looking colonic mucosa. The last colonoscopy, three and a half years after initiation of antirejection therapy, showed only lymphonodular hyperplasia without adenomatous changes (Figure 2B).

\section{Discussion}

We present a case of complete regression of colonic polyps in a patient with FAP treated with tacrolimus and MMF.

Tacrolimus is a macrolide antibiotic with immunomodulatory properties. It binds to FK506 binding protein FKBP12 and this complex inhibits calcineurin phosphatase, which, in turn, leads to inhibition of Ca-dependent events, such as interleukin-2 secretion, activation of nitric oxide synthase, and cell degranulation (7). Tacrolimus is widely used for prevention of rejection following kidney transplantation (8).

Immunosuppressive therapy is known to increase the general risk of malignancy, whereas tacrolimus use has particularly been linked to augmented risk of solid cancer $(9$, 10). Accordingly, the use of immunosuppressive agents in patients with cancer or precancerous lesions should be avoided due to their cancer-promoting effect. In our case, the use of tacrolimus was mandatory in order to suppress the rejection of the kidney transplant.

Notwithstanding our initial, well-founded concern, tacrolimus and MMF treatment of our patient resulted in rapid regression of multiple colonic polyps that had been observed (and partially removed) during previous colonoscopies prior to kidney transplantation. 

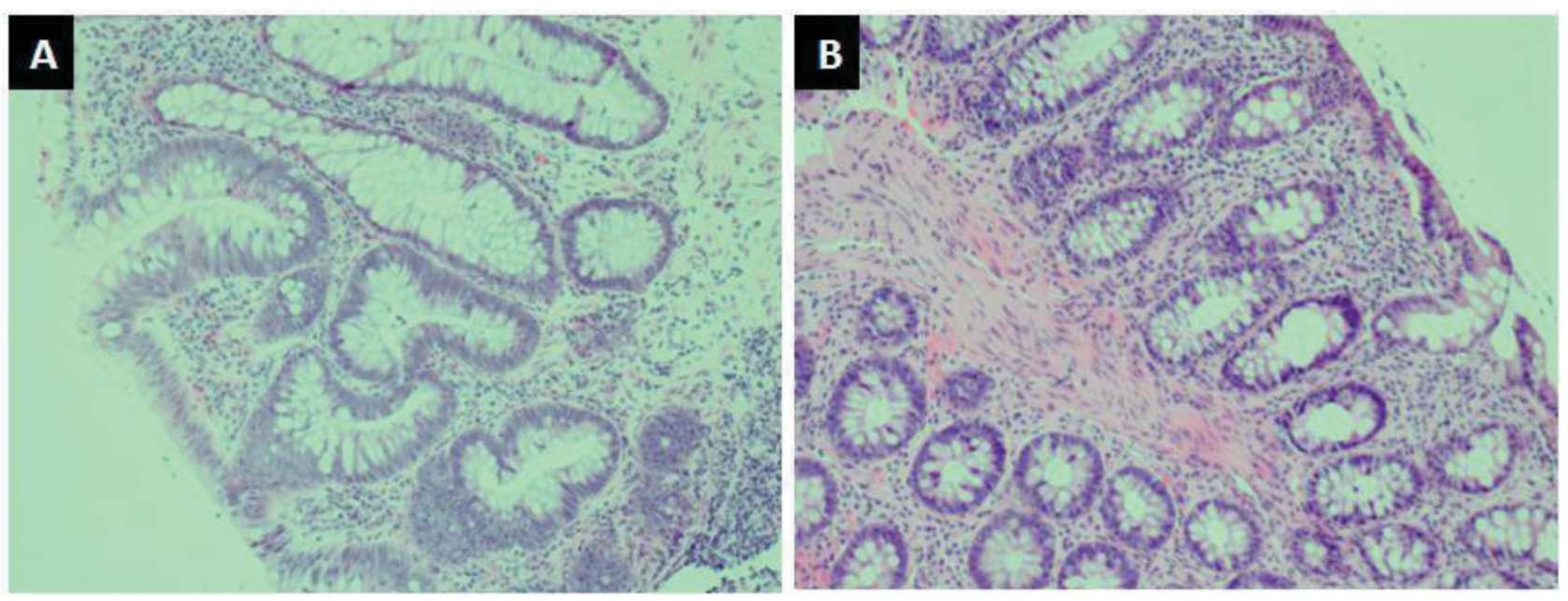

Figure 2. A: Pathological specimen at diagnosis: tubular adenoma with low-grade dysplasia. B. Pathological specimen obtained after three and a half years of tacrolimus and mycophenolate mofetil treatment, showing colonic mucosa without dysplasia. Hematoxylin-eosin staining, original magnification $\times 100$.

It is well known that patients with FAP have an almost $100 \%$ potential to develop colonic carcinoma by the age of 40 to 50 years (11). Attenuated FAP, observed in our patient (as defined by later onset and relatively small number of colonic polyps, fewer than 100) is less aggressive but still inevitably progresses to colorectal carcinoma development (11). We also know from our patient's family history that his family members with the same mutation had polyps early in life; the patient's grandmother died from metastatic colonic carcinoma, a younger relative developed fatal desmoid tumor after colectomy. These data indicate aggressive nature of this particular nonsense APC mutation.

This unanticipated absence of predicted aggressive course of FAP in our patient following initiation of immunomodulatory treatment prompted us to examine in detail the mode of action of tacrolimus and MMF in order to find a plausible mechanistic explanation for this intriguing clinical finding.

The nuclear factor of activated T-cell (NFAT) protein family was initially discovered as key regulators of interleukin-2 secretion and are currently known as targets of tacrolimus and cyclosporine A immunomodulatory action via calcineurin inhibition (12). The ability of tacrolimus to inhibit NFAT signaling with subsequent down-regulation of expression of oncogenic c-FOS and microphthalmiaassociated transcription factor (MITF) proteins is well known and might present a possible mechanism explaining the chemopreventative action of tacrolimus observed in our patient (13). It is worth mentioning that MITF is known to interact with $\beta$-catenin and to diversify the output of canonical WNT signaling, with subsequent enhancement of the repertoire of genes regulated by $\beta$-catenin (14). Given the fact that NFAT is a component of non-canonical (i.e. not mediated by $\beta$-catenin) WNT signaling pathway, one can entertain the possibility that inhibition of non-canonical WNT might, at least partially, counteract the constitutive activation of canonical WNT seen in FAP (15).

Recently, truncated fragments of the APC protein observed in colorectal cancer, accumulated in the cytoplasm as a result of nonsense mutations, have been shown to disrupt the orderly migration pattern of enterocytes, leading to overcrowding of intestinal crypts with precancerous cells (16). NFAT is a potential regulator of metastasis-associated transcriptional program and expression of NFATc1 has been associated with invasive behavior of colon cancer cells (17). Perhaps the potential ability of tacrolimus to impede local invasion of transformed colonocytes contributed to the regression of polyps in our patient.

Down-regulation of cell apoptosis is a well-known feature of colorectal carcinogenesis, while multiple chemoprevention modalities in colorectal cancer, such as use of non-steroidal anti-inflammatory drugs (NSAIDs) are aimed at restoration and even enhancement of apoptosis of colonic epithelial cells $(18,19)$. Multiple mechanisms have been implicated in this phenomenon (19). For our case, of particular interest is the observation of the anti-apoptotic effect of WNT5A-NFAT signaling (20). This observation, if coupled with the NFATinhibitory action of tacrolimus, and earlier report of Choi et al. on tacrolimus-induced apoptotic signal transduction pathway, might provide another putative mechanism of chemoprevention of tacrolimus in FAP, namely, enhancement of apoptosis in colonic mucosa (21). Furthermore, tacrolimus is known to induce increased secretion of transforming 
growth factor- $\beta$ (TGF $\beta$ ) in mammalian cells (22). Inactivation of TGF $\beta$ signaling, silencing its tumor suppressor activity, is of utmost importance in the development of colonic adenocarcinoma, whereas controlled release of exogenic TGF $\beta$ effectively impeded colon carcinogenesis in a murine model, as judged by significantly reduced number of aberrant crypt foci (precancerous lesions) and tumor number and size $(23,24)$.

One should also take into consideration the possible contribution of MMF to the observed attenuation of FAP phenotype. MMF is a potent inhibitor of inosine monophosphate dehydrogenase, the first enzyme in the de novo purine synthesis pathway. MMF treatment reduces guanine nucleotide pools in lymphocytes, with subsequent inhibition of activity of T- and B-lymphocytes resulting in immunosuppression (25). MMF is used extensively for posttransplantation immunosuppression and for treatment of autoimmune diseases, such as systemic lupus erythematosus and systemic sclerosis. It should be noted that MMF use following transplantation is not associated with an increased risk of malignancy (26). As well as its prominent immunosuppressive properties, MMF is endowed with tumorsuppressing capacity. The antitumor potential of nucleotide and nucleoside inhibitors of inosine monophosphate dehydrogenase was demonstrated three decades ago (27). To date, only scarce data referring to a putative anticancer effect of MMF are available. Reduction of inosine monophosphate dehydrogenase activity has been shown to potently inhibit endothelial cell proliferation in vitro and block tumor-induced angiogenesis in vivo (28). Furthermore, MMF is able to induce $\mathrm{G}_{1}$-S phase cell-cycle arrest and caspase-dependent apoptosis, as shown by alteration of mitochondrial membrane potential and cytochrome $c$ release followed by activation of caspases (29). Of note, the pro-apoptotic effect of MMF is synergistic to that of tacrolimus (21).

\section{Conclusion}

To the best of our knowledge, this is the first report on the ability of tacrolimus and MMF to impede development of colonic polyps in a patient with FAP. Several putative molecular mechanisms that might mediate this phenomenon, are available. Indeed, a recent study of Kawara et al., who demonstrated an anti-tumorigenic effect of tacrolimus and cyclosporine A on bladder cancer cell lines and in mouse xenograft model, coupled with the evidence that the antitumorigenic effect is modulated by NFAT inhibition provides robust support for our mechanistic interpretation of the clinical observation (30). Furthermore, in a recent study, Safaeian et al. analyzed the risk of colorectal cancer in US patients who underwent solid organ transplantation and did not observe elevated incidence of colorectal cancer in patients treated with tacrolimus and MMF, as opposed to patients treated with cyclosporine A and azathioprine posttransplantation (31). These findings are consonant with our conclusions. Further studies, aimed at deciphering the molecular events in the colonic mucosa of our patient and in untreated patients with FAP are underway. Our observation, if confirmed on larger number of patients, might provide the basis for the development of novel colorectal cancer prevention and treatment modalities aimed at NFAT inhibition and enhancement of apoptosis of colonic cells.

\section{Conflicts of Interest}

The Authors have stated that they have no conflict of interest in regard to this work.

\section{Acknowledgements}

The Authors express their gratitude to Professor Sergio A. Lamprecht for critical reading of the manuscript and for his insightful comments.

\section{References}

1 Vasen HFA, Möslein G,Alonso A, Aretz S, Bernstein S, Bertario L, Blanco I, Bülow S, Burn J, Capella G, Colas C, Engel C, Frayling I, Friedl W, Hes FJ, Hodgson S, Järvinen H, Mecklin JP, Møller P, Myrhøi T, Nagengast FM, Parc Y, Phillips R, Clark SK, Ponz de Leon M, Renkonen-Sinisalo L, Sampson JR, Stormorken A, Tejpar S, Thomas HJW and Wijnen J: Guidelines for the clinical management of familial adenomatous polyposis (FAP). Gut 57: 704-713, 2008.

2 Syngal S, Brand RE, Church JM, Giardiello FM, Heather L Hampel HL and Burt RW: ACG clinical guideline: genetic testing and management of hereditary gastrointestinal cancer syndromes. Am J Gastroenterol 110: 223-262, 2015.

3 Anastas J and Moon R: WNT signaling pathways as therapeutic targets in cancer. Nature Rev Cancer 13: 11-26, 2013.

4 Tsioulias G, Go MF and Rigas B: NSAIDS and colorectal cancer control: promises and challenges. Curr Phamacol Rep 1: 295-301, 2015.

5 Kawamori T, Lubet R, Steele VE, Kelloff GJ, Kaskey RB, Rao CV and Reddy BS: Chemopreventive effect of curcumin, a naturally occurring anti-inflammatory agent, during the promotion/ progression stages of colon cancer. Cancer Res 59: 597-601, 1999.

6 Lamprecht SA and Lipkin M: Chemoprevention of colon cancer by calcium, vitamin D and folate: molecular mechanisms. Nature Rev Cancer 3: 601-614, 2003.

7 Thomson A, Bonham C and Zeevi A: Mode of action of tacrolimus (FK506): molecular and cellular mechanisms. Ther Drug Monit 17: 584-91, 1995.

8 Muduma G, Hart W, Patel S and Odeyemi A: Indirect treatment comparison of belatacept versus tacrolimus from a systematic review of immunosuppressive therapies for kidney transplant patients. Curr Med Res Opin 23: 1-30, 2016.

9 Kopylov U, Vutcovici M, Kezouh A, Seidman E, Bitton A and Afif W: Risk of lymphoma, colorectal and skin cancer in patients with IBD treated with immunomodulators and biologics: A Quebec Claims Database study. Inf Bowel Dis 21: 1847-1853, 2015. 
10 Carenco C, Assenat E, Faure S, Duny Y, Danan G, Bismuth M, Herrero A, Jung B, Ursic-Bedoya J, Jaber S, Larrey D, Navarro $\mathrm{F}$ and Pageaux GP Tacrolimus and the risk of solid cancers after liver transplant: A dose effect relationship. Am J Transpant 15: 678-686, 2015.

11 Nieuwenhuis $\mathrm{MH}$ and Vasen HFA: Correlations between mutation site in APC and phenotype of familial adenomatous polyposis (FAP): A review of literature. Crit Rev Clin Oncol Hematol 61: 153-161, 2007.

12 Pan MG, Xiong Y and Chen F: NFAT gene family in inflammation and cancer. Curr Mol Med 13: 543-554, 2013.

13 Miyazaki M, Fujikawa Y, Takita $\mathrm{C}$ and Tsumura H: Tacrolimus and cyclosporine $\mathrm{A}$ inhibit human osteoclast formation via targeting the calcineurin-dependent NFAT pathway and an activation pathway for c-Jun or MITF in rheumatoid arthritis. Clin Rheumatol 26: 231-239, 2007.

14 Schepsky A, Bruser K, Gunnarsson GJ, Goodall J, Hallsson JH, Goding CR, Steingrimsson E, and Hecht A: The microphthalmia-associated transcription factor MITF interacts with $\beta$-catenin to determine target gene expression. Mol Cell Biol 23: 8914-8927, 2006.

15 Katoh M and Katoh M: WNT signaling pathway and stem cell signaling network. Clin Cancer Res 13: 4042-4045, 2007.

16 Nelson SA, Li Z, Newton IP, Fraser D, Milne RE, Martin DMA, Schiffmann D, Yang X, Dormann D, Weijer CJ, Appleton PL and Näthke IS: Tumorigenic fragments of APC cause dominant defects in directional cell migration in multiple model systems. Dis Models Mech 5: 940-947, 2012.

17 Tripathi MK, Deane NG, Zhu J, An H, Mima S, Wang X, Padmanabhan S, Shi Z, Prodduturi N, Ciombor KK, Chen X, Washington MK, Zhang B and Beauchamp DR: NFAT transcriptional activity is associated with metastatic capacity in colon cancer. Cancer Res 74: 6947-6957, 2014.

18 Bedi A, Pasricha P, Akhtar A, Barber JP, Bedi GC, Giardello FM, Zehnbauer BA, Hamilton SR and Jones RJ: Inhibition of apoptosis during development of colorectal cancer. Cancer Res 55: 1811-1816, 1995.

19 Chan T: Nonsteroidal anti-inflammatory drugs, apoptosis and colon-cancer chemoprevention. Lancet Oncol 3: 166-174, 2012.

20 Griesmann H, Ripka S, Pralle M, Ellenrieder V, Baumgart S, Buchholz M, Pilarsky C, Aust D, Gress TM and Michl P: WNT5A-NFAT signaling mediates resistance to apoptosis in pancreatic cancer. Neoplasia 15: 11-22, 2013.

21 Choi S, You H and Chung S: Tacrolimus-induced apoptotic signal transduction pathway. Transplant Proc 40: 2734-2736, 2008.
22 Ashwani H, Caims V and Hosenprud J: Tacrolimus induces increased expression of transforming growth factor- $\beta 1$ in mammalian lymphoid as well as nonlymphoid cells. Transplantation 67: 614-619, 1999.

$23 \mathrm{Yu} \mathrm{M}$, Trobridge P, Wang Y, Kanngurn S, Morris SM, Knoblauch $S$ and Grady WM: Inactivation of TGF- $\beta$ signaling and loss of PTEN cooperate to induce colon cancer in vivo. Oncogene 33: 1538-1547, 2014.

24 Mikhailowski R, Shpitz B, Polak-Charcon S, Kost Y, Segal C, Fich A and Lamprecht SA: Controlled release of TGF- $\beta$ impedes rat colon carcinogenesis in vivo. Int J Cancer 78:618-623, 1998.

25 Allison A and Eugui E: Mycophenolate mofetil and its mechanisms of action. Immunophamacol 47: 85-118, 2000.

26 Robson R, Cecka J, Opelz G, Budde M, Sacks S: Prospective registry-based observational cohort study of the long-term risk of malignancies in renal transplant patients treated with mycophenolate mofetil. Am J Transplant 5: 2954-2960, 2005.

27 Robins R: Nucleoside and nucleotide inhibitors of inosine monophosphate (IMP) dehydrogenase as potential antitumor inhibitors. Nucleosides Nucleotides 1: 35-44, 1982.

28 Chong C, Qian D, Pan F, Wei Y, Pili R, Sullivan DJ and Liu JO: Identification of type 1 inosine monophosphate dehydrogenase as an antiangiogenic drug target. J Med Chem 49: 2677-2680, 2006.

29 Takebe N, Cheng X, Fandy T, Srivastava R, Wu S, Shankar S, Bauer K, Shaughnessy J and Tricot G: IMP dehydrogenase inhibitor mycophenolate mofetil induces caspase-dependent apoptosis and cell cycle inhibition in multiple myeloma cells. Mol Cancer Ther 5: 457-466, 2006.

30 Kawahara T, Kashiwagi E, Li Y, Zheng Y, Miyamoto Y, Netto GI, Ishiguro $\mathrm{H}$ and Miyamoto $\mathrm{H}$ : Cyclosporine $\mathrm{A}$ and tacrolimus inhibit urothelial tumorigenesis. Mol Carcinogen 55: 161-169, 2016.

31 Safaeian M, Robbins H, Berndt S, Lynch CF, Fraumeni JF and Engels EA: Risk of colorectal cancer after solid organ transplantation in the United States. Am J Trasplant 16: 960-967, 2016.

Received April 17, 2017

Revised April 27, 2017

Accepted May 2, 2017 